\title{
BAHIA BLANCA Y SU ZONA DE INFLUENCIA
}

\section{SU POSICION GEOGRAFICA}

Se halla ubicada al sud de la Provincia de Buenos Aires, sobre el Océano Atlántico, en una bahía natural, que le permite tener un gran puerto con una profundidad de 30 pies, al que pueden llegar los grandes transatlánticos, uno de los pocos grandes puertos naturales del país, que naturalmente debiera ser la salida obligada al exterior de su "hinterland".
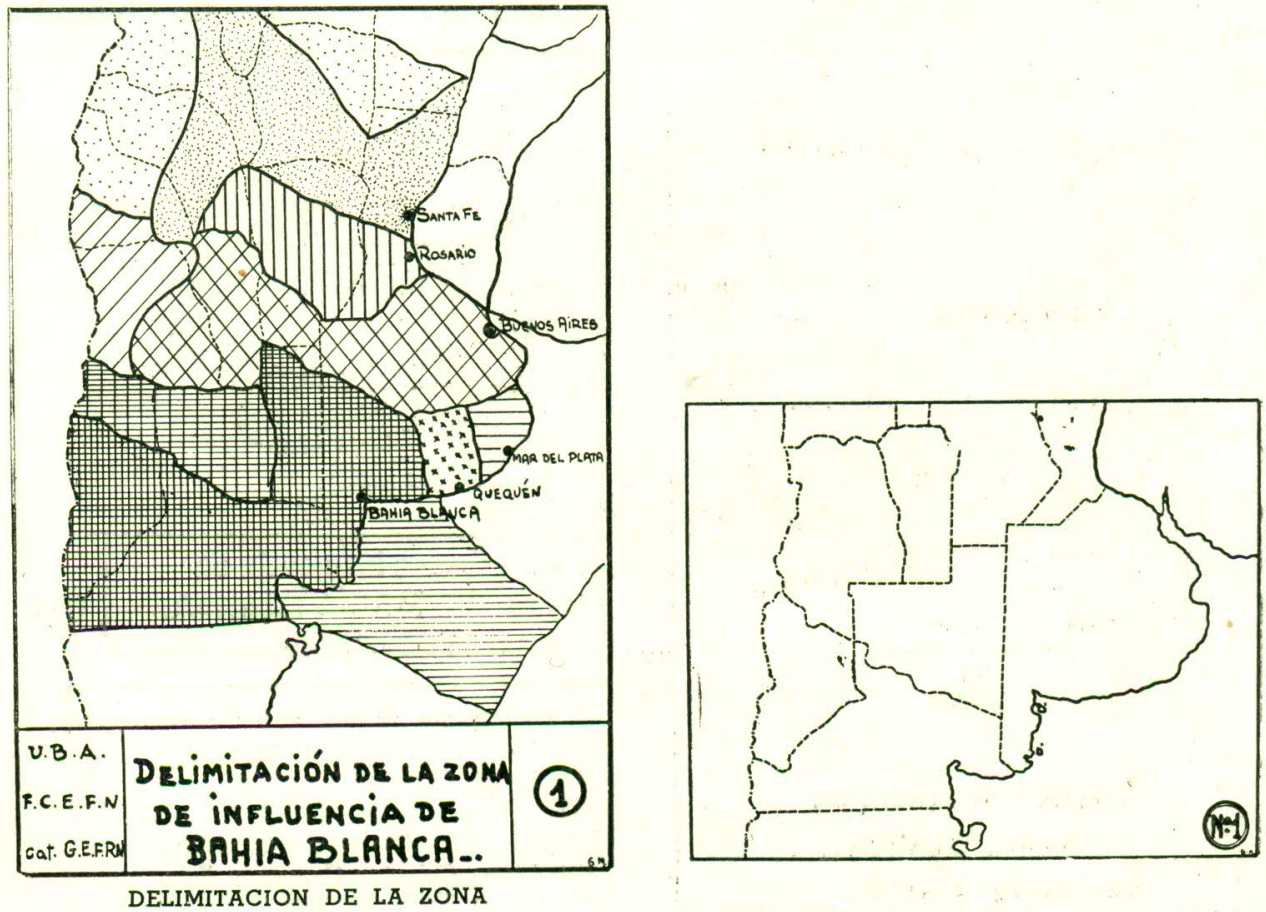

\footnotetext{
Mendoza (parte) ... $50.000 \mathrm{Km}^{2}$.

Neuquén ......... $94.000 \mathrm{Km}^{2} .90 .000 \mathrm{Hab}$.

Río Negro ..........203.000 $\mathrm{Km}^{2}$. $140.000 \mathrm{Hab}$

y S. Luis $143.000 \mathrm{Km}^{2} . \quad 170.000 \mathrm{Hab}$

La Pampa Y S. Luis $143.000 \mathrm{Km}$

.

Plafond Submarino. $230.000 \mathrm{Km}^{2}$

Potlación total ......... 1,000.000 Hab.tantes

Extensión total ......... $650.000 \mathrm{Km}^{2}$.

Elensión $230.000 \mathrm{Km}^{2}$
} 
No obstante que se trata de una población nueva, fundada en 1828 en momentos que el país estaba en armas, como una necesidad militar para asegurar el dominio del lejano sud y como eslabón de unión a Carmen de Patagones que ya existía medio siglo antes, el determinismo geográfico lleva a Bahía Blanca a pasar a primer rango, y transformarse en el núcleo humano más importante del sud.

\section{DESCRIPCION DE LA ZONA DE INFLUENCIA}

Esta zona de influencia se halla limitada por la de los puertos de QUEQUEN y BUENOS AIRES. Es conjuntamente con la de Santa Fe, una de las zonas más extensas; comprende (ver lámina 1) más de la quinta parte de la superficie del país, 21,5\%, y dentro de esta extensión tiene un $20 \%$ de la Pampa húmeda templada que es la zona de los grandes cultivos extensivos, y de las praderas naturales, donde se encuentran las $3 / 4$ partes de la riqueza del país. Es ésta la "Argentina Rica", como la han definido algunos autores.

Complementa esta zona, gran parte de la Gobernación de la PAMPA, todo el RIO NEGRO, y NEUQUEN, y en el caso de navegarse el río Colorado,

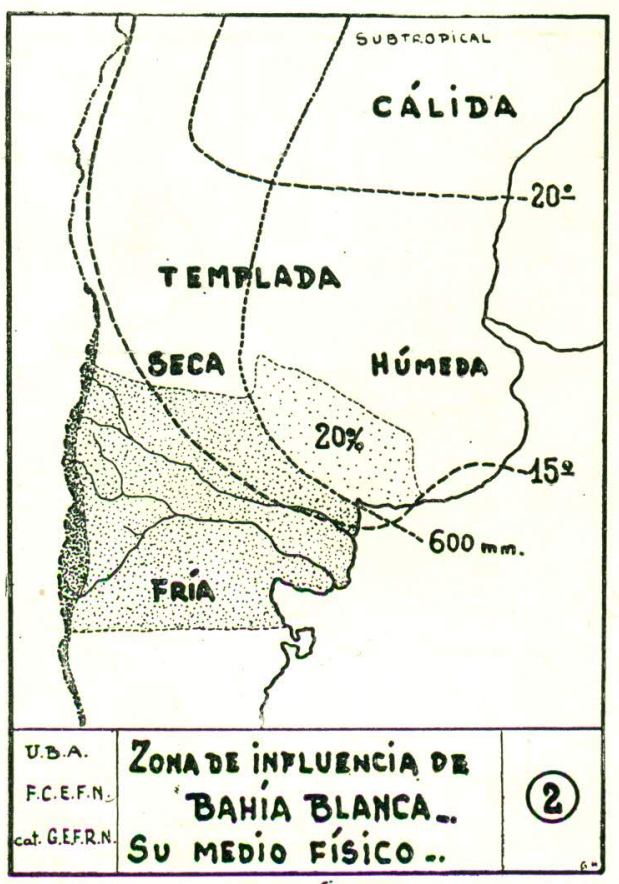

SU MEDIO FISICO

ZONA SUBTROPICAL (lim. Z'OO)

ZONA TEMPLADA. (lim. $\left.15^{\circ}\right)$

Seca

Húmeda

$20 \%$ zona templ. y húmeda R. A. FRIA

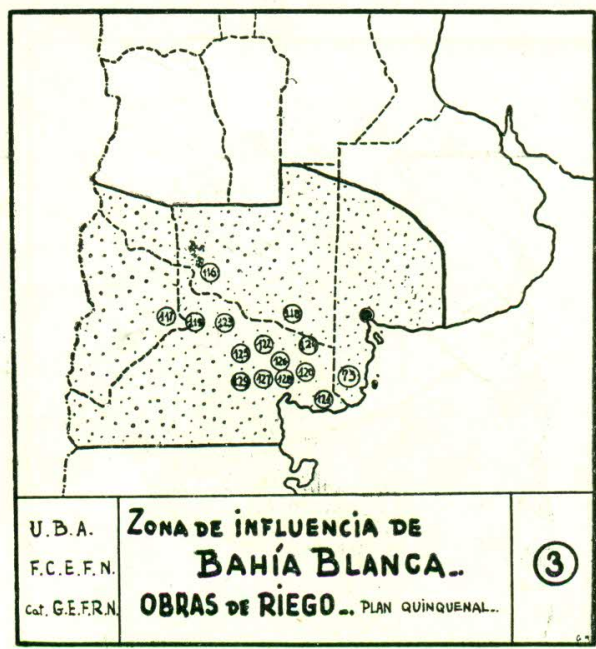

OBRAS DE RIEGO PLAN OUINOUENAL

116 Riego Colonia 25 de Mayo.

117 Riego Chacras Neuquén.

118

119 Desagües Río Negro Superior.

120 Riego Colonias Conesa y Fría

121 Riego Valle Viedma.

19? Riego Isla Choele Choel.

123

4 Riego Río Colorado y E. Bustos.

Canal Choele Choel San Antonio Oeste.

Canal Valle Medio San Antonio Oeste.

Canal Río Negro a San Antonio Oeste.

Riego Chimpay y Belisle.

Riego Valle Medio Río Negro. 
toda la Gobernación de la Pampa y el sud de Mendoza (zona del Río Grande) se incorporaría también a esta región. No obstante tratarse de una región seca, cuenta con dos grandes ríos, el Negro y el Colorado y sus afluentes, con valles en parte ya irrigados de explotación agrícola intensiva, y con proyecciones a un desarrollo muy grande, como puede observarse en la lámina 3, con las obras de Riego que se prevén en el plan Quinquenal (primero).

La primera región (pampa húmeda) le da a Bahía Blanca, una tercera parte de la producción de trigo (de la Nación) conjuntamente con el centro de gravedad de una de las zonas de mayor intensificación de estos cultivos, y que coincide también con la zona de las mayores explotaciones de ganado ovino. El hinterland de Bahía Blanca cuenta con el $25 \%$ de esta riqueza (ganado lanar).

Aparte de estas dos riquezas que son las básicas de la región, y que le dan su característica típica, tiene el $14 \%$ de la fruta fresca en el valle del Río Negro Superior, y el $9 \%$ del tomate, etc.

Cuenta con las salinas en explotación más importantes del país; en materia de asfaltitas, es el principal centro que tenemos en la República, y se explotan; cuenta también con una reserva de yacimientos de carbones subituminosos; con grandes depósitos de yeso en parte en explotación; con yacimientos de sal de roca, y azufre, con filones de oro y oro aluvional (re-

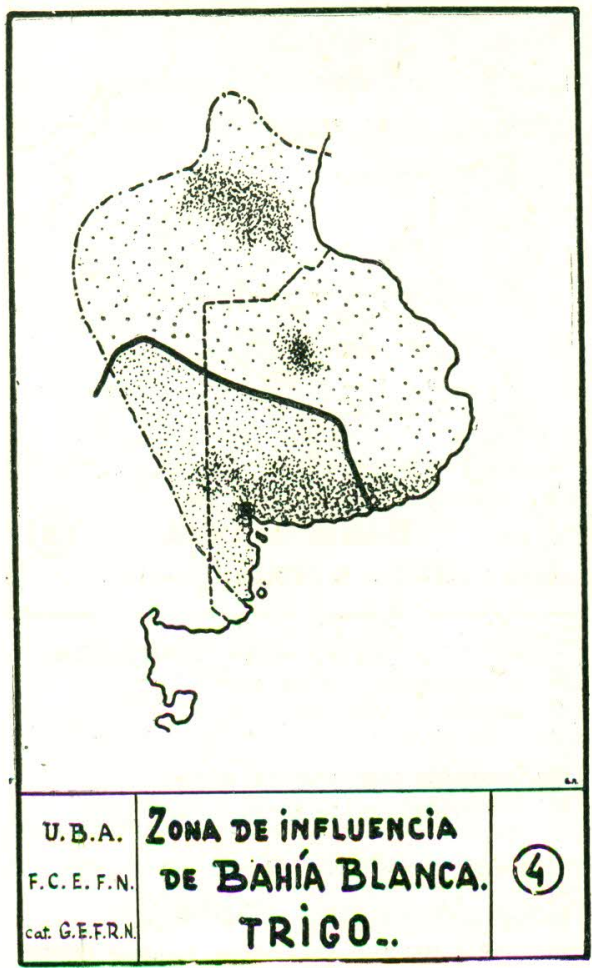

TRIGO

Zona de B. Blanca aprox. 1/3 de la Producción. $3 / 3$ de la Producción - 6,5 millones de T.

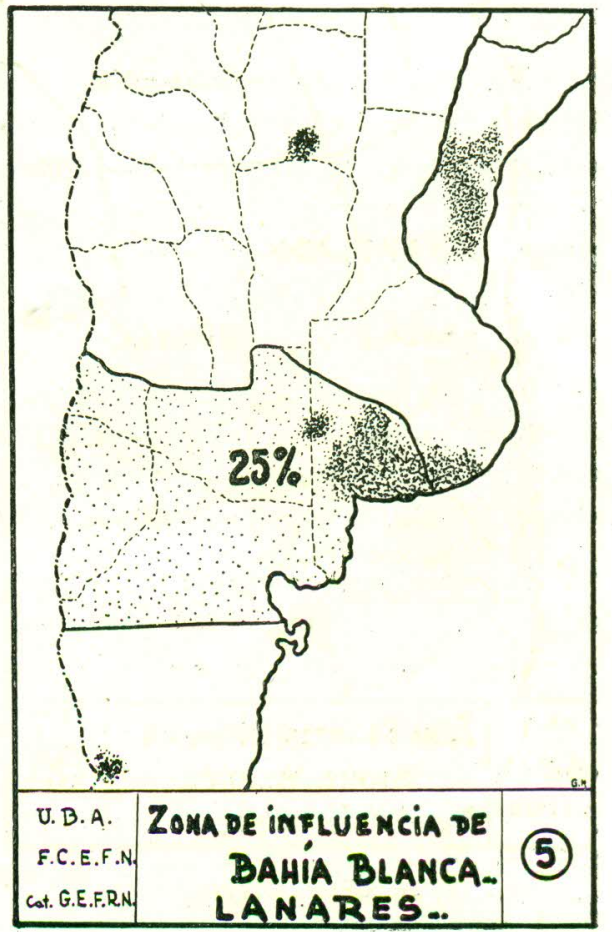

LANARES

Zona de Influencia de Bahía Blanca $25 \%$ 
gión de Andacollo). Con depósitos de petróleo importantes (5\% de la producción del país), con yacimientos de cobre, plomo, zinc, estaño y plata, y con la zona ferrífera más interesante para las grandes fundiciones del país en Sierra Grande, donde veremos instalarse la gran industria pesada que nuestra Patria tanto necesita para construir su propio plantel industrial y asegurar su defensa.

Tiene también como complemento de todas las riquezas enumeradas, la cuarta parte del plafond submarino, con posibilidades de pesca muy grandes.

Como si todo fuera poco, dispone de una zona de bosques grande, y un conjunto de usinas hidroeléctricas que prevé su ejecución del Plan Quinquenal (primer) que le dan a Bahía Blanca, una posición del todo privilegiada en el conjunto de la Argentina.

No olvidemos del gasoducto, que en más de $1 / 3$ de su recorrido, pasa por esta zona y de la enorme cuenca de agua caliente $\left(61^{\circ}\right)$ que se presenta a 700 m. de profundidad.

\section{LOS MEDIOS DE TRANSPORTE}

En cuanto a los medios de comunicación, este extenso territorio, se mantiene siempre en posición de privilegio.

En materia de ferrocarriles: Toda la región de la provincia de Buenos Aires está perfectamente atendida por un conjunto de ramales que va a toda esta zona, con un predominio del todo marcado sobre otros medios de transportes. El antiguo Bahía Blanca y Nordoeste le ha dado salida a toda la parte rica de la Gobernación de La Pampa y los ramales a Zapala y Bariloche le han asegurado su predominio en Río Negro y Neuquén.

El Plan Quinquenal prevé la ampliación de las vías de Contralmirante Cordero a Chos Malal y su unión a Bardas Blancas, las que le aseguran su vinculación al sur de Mendoza. Otro proyecto del mismo Plan presenta la continuación del ramal de Zapala a Chile, lo que aumentaría en forma muy apreciable los mercados de Bahía Blanca.

Referente a caminos: El solo hecho de observar la lámina 12 que se refiere a las obras viales del Plan Quinquenal, permite observar todo un emparrillado que le asegura todos los accesos al amplio territorio.

Los ríos: Como si no fuesen suficientes todos los medios de transporte que ya hemos comentado, cuenta esta región con dos grandes ríos, el Negro y el Colorado. El primero ya nadie duda de su navegabilidad, aunque su tráficc carece por ahora de importancia, en cambio al segundo, no obstante tener grandes posibilidades su navegación, con barcazas de poca profundidad, hasta el momento nada se hace, a pesar de que antes, hace más de medio siglo, se usaba como medio de comunicación (Navegación interior del país por el Ing. Luis A. Huergo). Personalmente he comprobado sus grandes 


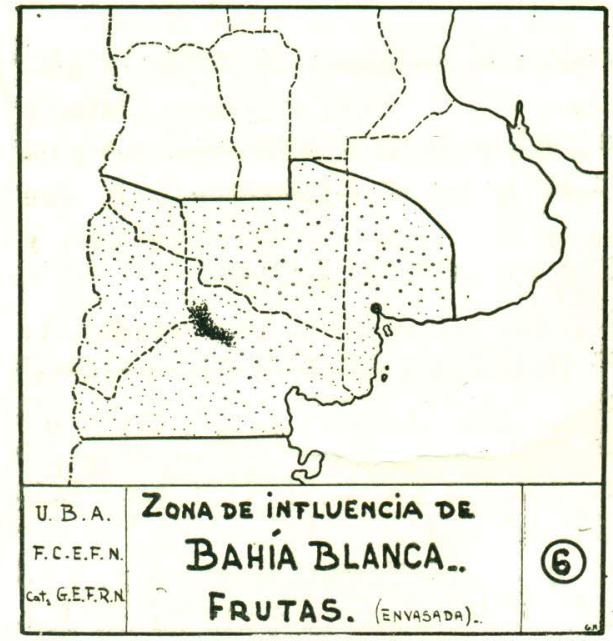
FRUTAS

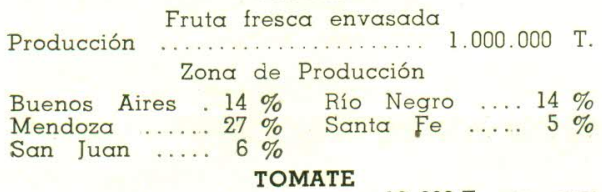

Producción $\ldots . . \ldots \ldots \ldots \ldots . \quad 10.000 \mathrm{~T}$. conserva Zona àe Producción

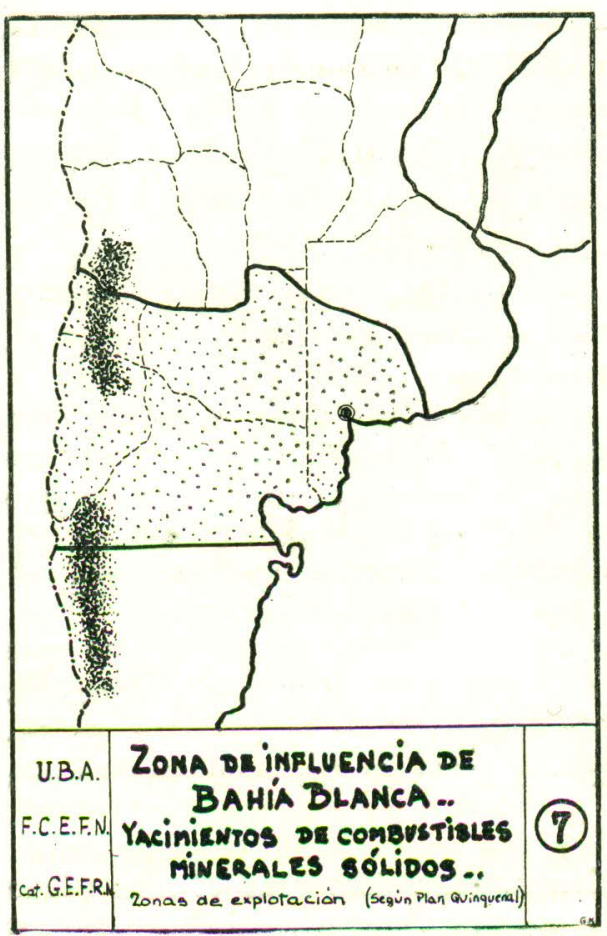

Yacimientos de combustibles minerales sólidos. Zonas de explotación según Plan Quinquenal.

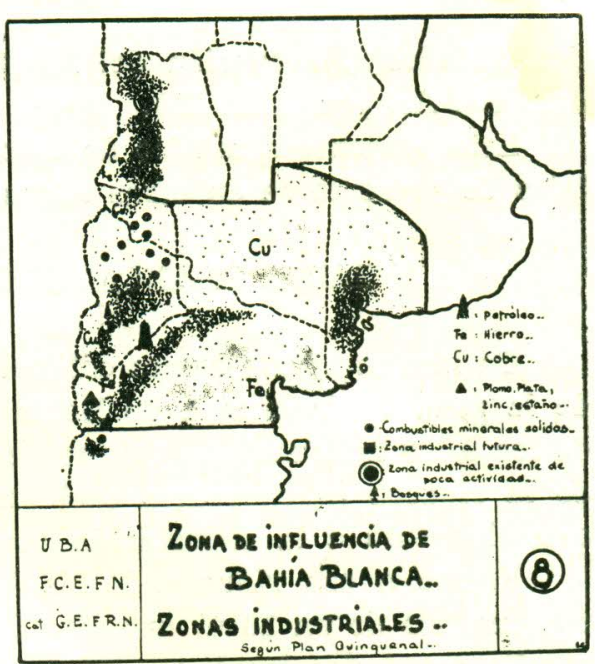

ZONAS INDUSTRIALES SEGUN PLAN

Fe. Hierro.

Cu. Cobre.

Plomo; plata; zinc; estado.

Combustibles minerales sólidos.

bosques.

Petróleo.

Zona negra: Zona industrial existente de poca actividad

Zona amarilla: ona industrial futura.

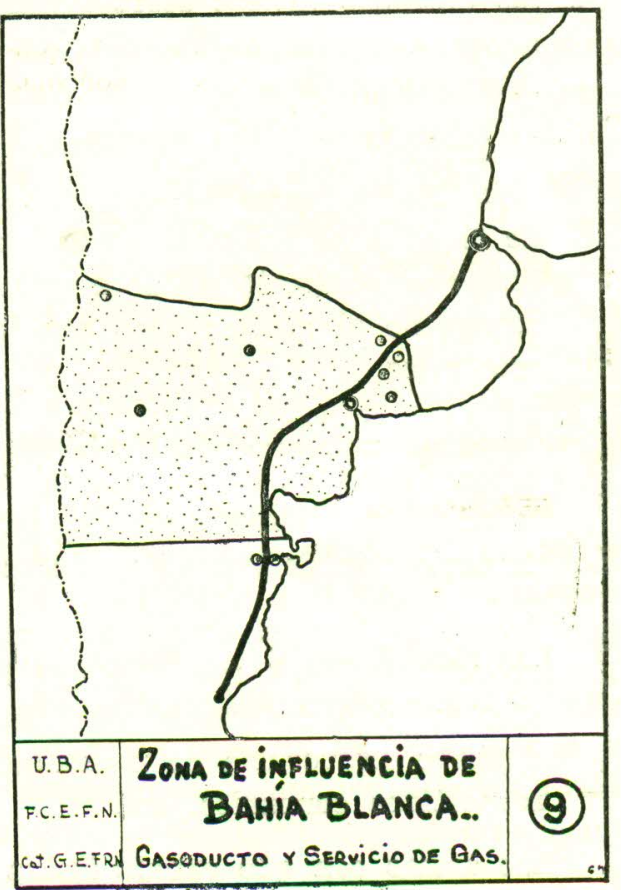

Gasoducto y servicio de gas según 
posibilidades (Navegación del Río Colorado - $2^{\text {o }}$ Congreso Industrial y Minero Bs. As. por G. P. H.).

Este conjunto armónico de todos los medios de comunicación, está muy lejos de presentarse en otras zonas económicas de la Argentina.

\section{BAHIA BLANCA FRENTE A SU HINTERLAND}

En lámina aparte hemos estimado los réditos que representan la riqueza de la zona de influencia de Bahía Blanca llegando a una cifra del órden de los 3.000 millones de pesos moneda nacional, con un predominio absoluto de la riqueza agropecuaria casi las tres cuartas partes, y ésta vinculada extrechamente a la exportación (economía colonial).

Frente a esa riqueza, Bahía Blanca está prácticamente ausente, sólo se beneficia de un $13 \%$ aproximadamente, es decir que se trata de un desarrollo económico local, que no está en la escala de su zona económica, de la que prácticamente en nada participa.

\section{RESPECTO A LA CENTRALIZACION DE BUENOS AIRES}

Entre las muchas explicaciones que podrían presentarse y las que nos interesan observar, son las que siguen:

$1^{\circ}$ las antiguas tarifas ferroviarias competitivas y las de tráfico común que aunque derogadas en parte el 1-2-49 aún persiste su efecto, como fuerza de inercia.

$2^{\circ}$ la tendencia hacia las grandes embarcaciones marítimas de $30^{\prime}$ de calado. No obstante que Arroyo Pareja y Puerto Militar tienen 30 pies de calado, los puertos comerciales de Ing. White y Galván tienen 25. Sobre este aspecto nada se opone a un racionamiento de las bodegas, semejante al que se hace con los vagones de distintas capacidad ferroviarios.

$3^{0}$ La centralización administrativa de Buenos Aires. Si desde Londres no había dificultad en manejar nuestros ferrocarriles, no es posible que la administración a distancia nos sea un tropiezo difícil de salvar. En Organización Industrial verán la solución de esta difícil situación.

$4^{\circ} \mathrm{La}$ centralización comercial (mercados). Un principio económico que no admite dudas, nos dice que la creación de la demanda provoca la oferta; es decir que la planificación industrial, y la creación de industrias, provocaría de inmediato la aparición de los mercados de sus respectivos consumos, etc.

Las armas con que cuenta Bahía Blanca, para descontar el más pleno éxito de esta reacción, son la utilización de las fuentes de energía hidroeléctrica, y térmicas (agua caliente), la economía de vagones con el uso de 


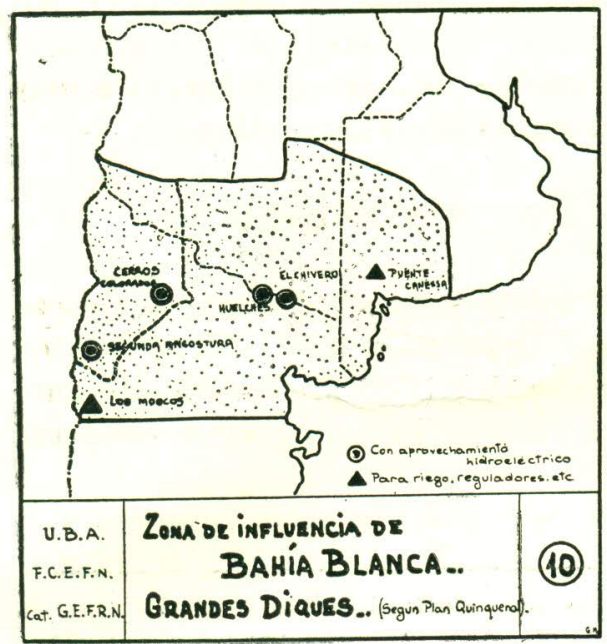

GRANDES DIQUES SEgUN PLAN QUINQUENAL

Con aprovechamiento hidroeléctrico. Segunda Angostura; Cerros Colorados; Huelches; El Chivero.

Para riego, reguladores, etc.: Puente Canessa: Los Moscos.

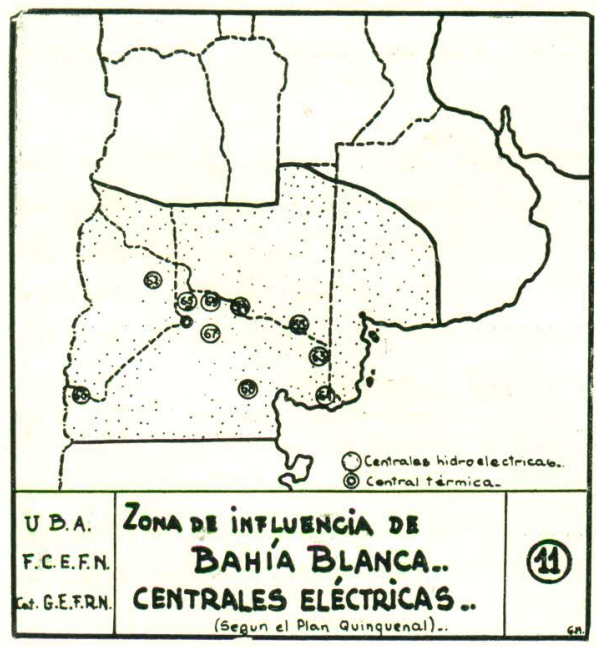

CENTRALES ELECTRICAS SEGUN PLAN QUINQUENAL.

Centrales Hidroeléctricas: 54, Huelches; 55, El Chivero. 60. Segunda Angostura: 62, Cerros Colorados: 63 Rápidos Andersen: 64, Canal Principal Valle Viedma. 65 Canal Principal Río Negro Superior; 66, id. id.; 67, Canal Matriz Valle Medio; 68, Canal San Antonio Oeste; 69, Canal Principal Río Negro Superior.

Centrales Térmicas.

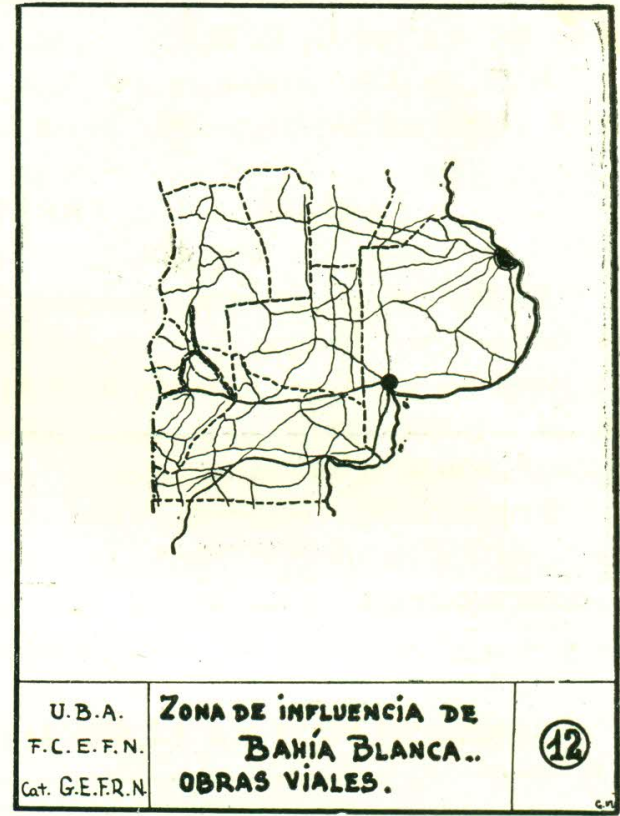

Obras Viales según Plan Quinquenal. Ampliación de puertos según Plan Quinquenal.

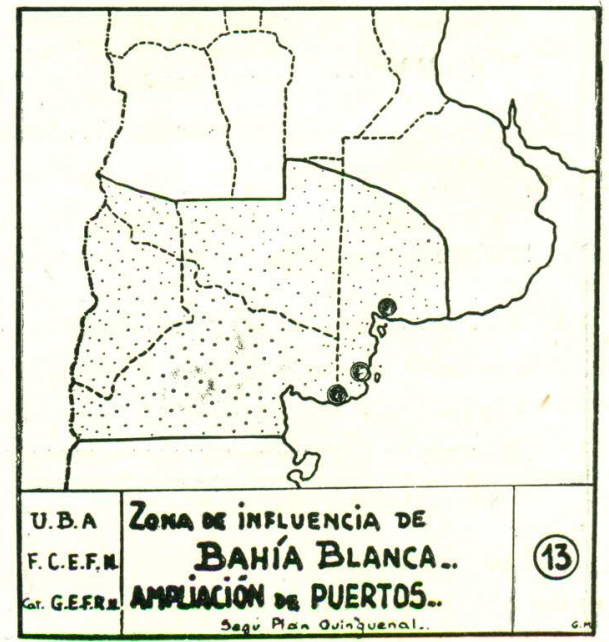



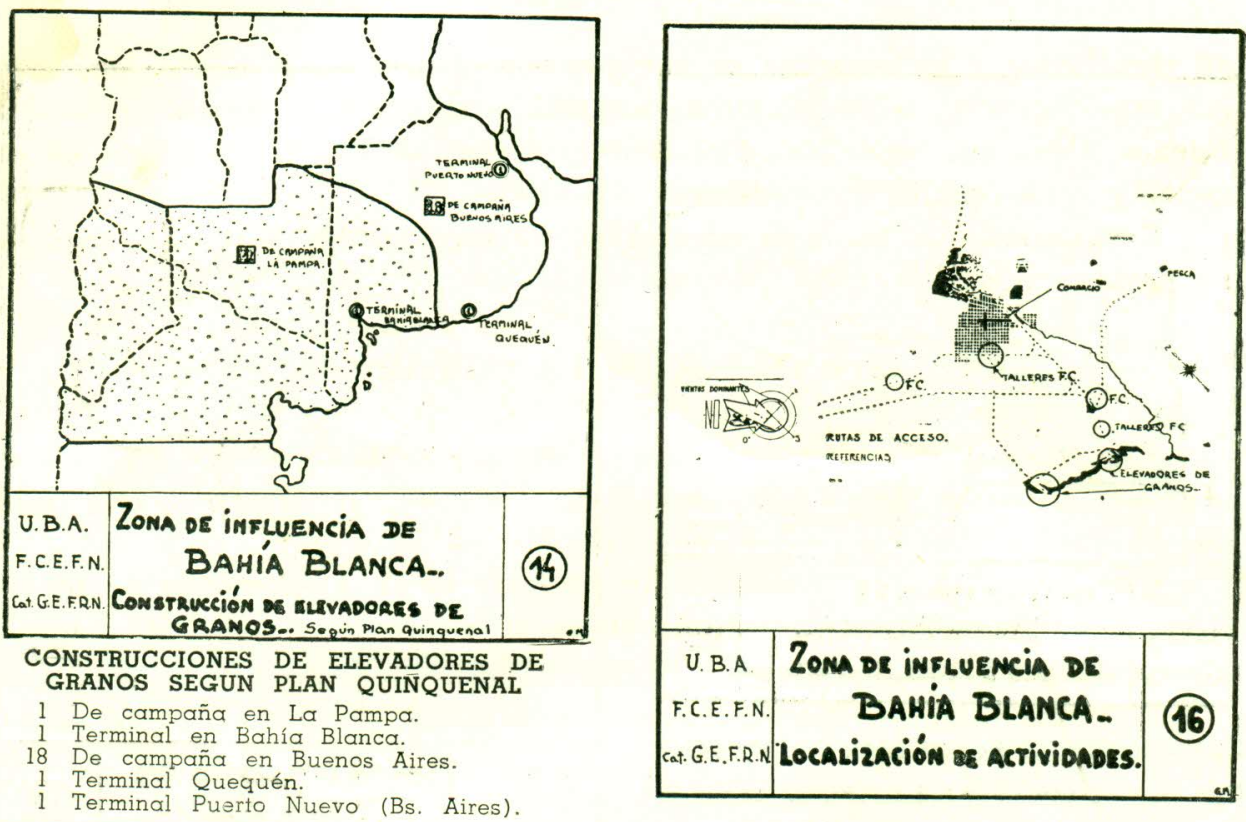

\section{REDITOS QUE PRODUCE LA ZONA}

AGRICULTURA:

(Cólculo estimativo)

l/3 Trigo x 6,5 millones x $200 \$ / \mathrm{T}$.

Le corresponde a Bahía Blanca como produc

Le corresponde a Bahía Blanca como comerc.

Demás cereales

\begin{tabular}{rr}
440 & 50 \\
& 20 \\
50 & 15 \\
\hline 490 & -85 \\
\hline
\end{tabular}

GANADERIA:

1/4 ovinos x 320 millones $\mathrm{kg} . \mathrm{v} 2 \$ / \mathrm{k}$.

$1 / 4$ lana 250 mil T. $x 15.000 \$ / T$.

Id. cueros 50 millones $\mathrm{n}^{0} \times 40$

Demas

TOTAL

\begin{tabular}{rr}
160 & 40 \\
950 & \\
500 & 50 \\
100 & \\
\hline 1.710 & 90 \\
\hline
\end{tabular}

PESCA:

4 millon $\in \mathrm{s}$ kg. $\times 2 \% / \mathrm{kg}$. INDUSTRIA:

Zona Buenos Aires

La Pampa, R. Negro; Neuquén

\begin{tabular}{ll}
250 & 200 \\
$\frac{150}{408}$ & -208 \\
\hline 3.000 & $\frac{1}{400}=13 \%$
\end{tabular}

TOTAL GENERAL

TOT'AL

U. B. A.

F.C.E.F.N.

Cat.

G.E.F.R.N.
ZONA DE INFLUENCIA DE BAHIA BLANCA

REDITOS QUE PRODUCE

15 
su red fluvial, y la economía de vagones con el uso de su puerto, desviando las exportaciones hacia su curso natural, evitándose el desplazamiento a Buenos Aires que significan $700 \mathrm{Km}$. innecesarios, con la economía de vagones y combustibles consiguientes.

Explotando esta posición económica, no será posible que se le succione su propia riqueza.

\section{ANALISIS DE LA CIUDAD}

Su principal actividad es la comercial, que se lleva a cabo en su zona céntrica, alrededor de su plaza principal, y en las cuatro principales calles que se unen a ella.

Las industrias que todas son de carácter local, a excepción de los talleres del ferrocarril, se vinculan a la construcción, al transporte, a la alimentación $\mathrm{y}$ al vestido.

El puerto, que ha sido concebido con carácter regional, en la actualidad es un recuerdo del pasado.

\section{SUS POSIBILIDADES DE DESARROLLO}

Frente a este panorama, mucho es lo que el país espera de Bahía Blanca, no obstante haber alcanzado el primer rango de las poblaciones del sud, debe todavía integrar su proceso de evolución, con la producción industrial, aprovechando las riquezas de su zona, para el mejor desarrollo económico del país, y para brindarle (al país) una parte de sus abastecimientos a los menores costos y un mercado de mano de obra descentralizada, que irá cimentando la argentinidad en nuestra Patagonia, por tanto tiempo olvidada. 